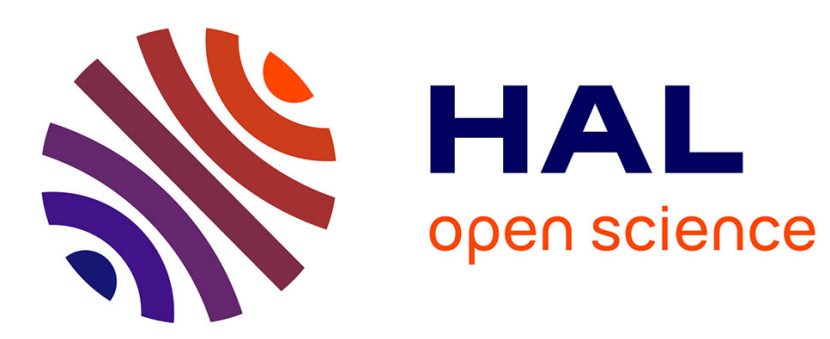

\title{
Le décentrement du regard géographique dans le Shuijing zhu de Li Daoyuan († 527)
}

Alexis Lycas

\section{To cite this version:}

Alexis Lycas. Le décentrement du regard géographique dans le Shuijing zhu de Li Daoyuan († 527). Bulletin de l'Ecole française d'Extrême-Orient, 2018, 104 (1), pp.241-266. 10.3406/befeo.2018.6273 . halshs-02308336

\section{HAL Id: halshs-02308336 \\ https://shs.hal.science/halshs-02308336}

Submitted on 10 Jan 2020

HAL is a multi-disciplinary open access archive for the deposit and dissemination of scientific research documents, whether they are published or not. The documents may come from teaching and research institutions in France or abroad, or from public or private research centers.
L'archive ouverte pluridisciplinaire HAL, est destinée au dépôt et à la diffusion de documents scientifiques de niveau recherche, publiés ou non, émanant des établissements d'enseignement et de recherche français ou étrangers, des laboratoires publics ou privés. 


\section{Le décentrement du regard géographique dans le Shuijing zhu} de Li Daoyuan († 527)

Alexis Lycas

\section{Citer ce document / Cite this document :}

Lycas Alexis. Le décentrement du regard géographique dans le Shuijing zhu de Li Daoyuan († 527). In: Bulletin de I'Ecole française d'Extrême-Orient. Tome 104, 2018. pp. 241-266;

doi : https://doi.org/10.3406/befeo.2018.6273

https://www.persee.fr/doc/befeo_0336-1519_2018_num_104_1_6273

Fichier pdf généré le 07/01/2020 


\title{
Résumé
}

Ouvrage fondamental mais méconnu de l'histoire et de la géographie de la Chine ancienne, le Shuijing zhu ou Annotations aux Itinéraires des cours d'eau a été compilé au début du Vle siècle par Li Daoyuan (mort en 527), un lettré chinois ayant servi la cour non chinoise des Wei du Nord. Le texte recèle nombre d'informations relatives aux fleuves et aux montagnes, aux villes et à leur organisation, mais encore aux cultes locaux, aux légendes régionales et aux personnages notables. Véritable compendium des savoirs, notamment techniques, le Shuijing zhu mesure les évolutions de l'espace chinois dans le temps et constitue en outre un réservoir de textes locaux qui auraient sans doute été perdus sans le travail de Li Daoyuan. Une fois l'auteur et son œuvre présentés, cet article analyse le développement de la littérature géographique à l'aune d'un thème particulier, celui du « décentrement » du regard géographique. Une seconde partie s'attache aux implications formelles d'un tel décentrement, à travers le choix de l'annotation, d'une architecture textuelle découlant des cours d'eau de l'empire et au-delà, et par la cohérence d'ensemble et la précision des descriptions hydrographiques.

\begin{abstract}
A fundamental but little known work on the history and geography of ancient China, the Shuijing zhu or Annotations to the Itineraries of Rivers was compiled at the beginning of the 6th century by Li Daoyuan (died 527), a Chinese scholar who served the non-Chinese court of the Northern Wei. The text contains much information about rivers and mountains, cities and their organisation, but also about local cults, regional legends and notable people. The Shuijing zhu is a true compendium of knowledge, especially technical. It also traces the evolution of Chinese landscape over time, and serves as a repository of local writings that would have been lost otherwise. After presenting the author and his text, the first part of the article analyses the development of geographical literature in light of a "decentering" of the geographical perspective. A second part focuses on the formal implications of such a decentering, through the choice of annotation, of a textual architecture derived from the rivers of the empire and beyond, and through the overall coherence and accuracy of hydrographic descriptions.
\end{abstract}




\title{
Le décentrement du regard géographique dans le Shuijing zhu de Li Daoyuan († 527)
}

\author{
Alexis LyCAS
}

\begin{abstract}
A fundamental but little known work on the history and geography of ancient China, the Shuijing zhu 水經注 or Annotations to the Itineraries of Rivers was compiled at the beginning of the 6th century by Li Daoyuan 麗道元 (died 527), a Chinese scholar who served the non-Chinese court of the Northern Wei. The text contains much information about rivers and mountains, cities and their organisation, but also about local cults, regional legends and notable people. The Shuijing zhu is a true compendium of knowledge, especially technical. It also traces the evolution of Chinese landscape over time, and serves as a repository of local writings that would have been lost otherwise. After presenting the author and his text, the first part of the article analyses the development of geographical literature in light of a "decentering" of the geographical perspective. A second part focuses on the formal implications of such a decentering, through the choice of annotation, of a textual architecture derived from the rivers of the empire and beyond, and through the overall coherence and accuracy of hydrographic descriptions.
\end{abstract}

Keywords: Shuijing zhu; Li Daoyuan; geography; Imperial China; Six dynasties; Buddhism.

\section{Résumé}

Ouvrage fondamental mais méconnu de l'histoire et de la géographie de la Chine ancienne, le Shuijing zhu 水經注 ou Annotations aux Itinéraires des cours d'eau a été compilé au début du vI siècle par Li Daoyuan 麗 道元 (mort en 527), un lettré chinois ayant servi la cour non chinoise des Wei du Nord. Le texte recèle nombre d'informations relatives aux fleuves et aux montagnes, aux villes et à leur organisation, mais encore aux cultes locaux, aux légendes régionales et aux personnages notables. Véritable compendium des savoirs, notamment techniques, le Shuijing zhu mesure les évolutions de l'espace chinois dans le temps et constitue en outre un réservoir de textes locaux qui auraient sans doute été perdus sans le travail de Li Daoyuan. Une fois l'auteur et son ouvre présentés, cet article analyse le développement de la littérature géographique à l'aune d'un thème particulier, celui du «décentrement» du regard géographique. Une seconde partie s'attache aux implications formelles d'un tel décentrement, à travers 
le choix de l'annotation, d'une architecture textuelle découlant des cours d'eau de l'empire et au-delà, et par la cohérence d'ensemble et la précision des descriptions hydrographiques.

Mots-clés : Shuijing zhu; Li Daoyuan; géographie; Chine impériale; Six dynasties; bouddhisme. 


\title{
Le décentrement du regard géographique dans le Shuijing zhu de Li Daoyuan (†527)
}

\author{
Alexis LyCAs*
}

\section{Introduction}

Selon Édouard Chavannes (1865-1918), le Shuijing zhu 水經注 (Annotations aux Itinéraires des cours d'eau) de Li Daoyuan 麗道元 (†527) 《est un document géographique de la plus haute importance, mais il est souvent fort obscur ${ }^{1} »$. Valable il y a plus d'un siècle, son jugement l'est encore aujourd'hui. En effet, si le Shuijing zhu est très fréquemment cité, que ce soit par des exégètes des siècles passés ou par des sinologues contemporains, il ne l'est bien souvent qu'à titre de simple complément, venant s'ajouter à d'autres ouvrages, index ou dictionnaires encyclopédiques. Le Shuijing $z h u$ est jugé trop volumineux, monolithique ou rébarbatif, et peu d'auteurs s'y sont intéressés autrement que comme ouvrage de référence ${ }^{2}$.

Or, le Shuijing zhu s'avère d'une importance certaine si l'on souhaite comprendre d'une part la manière dont l'espace est perçu et représenté par un auteur chinois du premier millénaire de l'ère impériale, et d'autre part l'évolution du savoir géographique (sources, outils, méthodes, enquêtes) en Chine durant sa période de formation. Le caractère rétrospectif de l'ouvrage met au jour la manière dont Li Daoyuan écrivait l'histoire et se représentait le monde dans lequel il évoluait, et, surtout, dont il était l'héritier. Traditionnellement, au sein de la production historiographique chinoise, les ouvrages géographiques présentent les configurations spatiales de l'Empire telles qu'elles étaient au temps de leur rédaction ou dans les textes dont ils s'inspirent ${ }^{3}$. C'est pourquoi l'intérêt des sinologues s'est généralement porté sur la géographie historique et ses conséquences dans le domaine de l'histoire politique ${ }^{4}$. Toutefois, la compréhension des dispositions spatiales

\footnotetext{
* Institut Max Planck d'histoire des sciences, Berlin, alycas@mpiwg-berlin.mpg.de. J'adresse mes remerciements les plus vifs à Marianne Bujard pour ses conseils et sa relecture, ainsi qu'aux évaluateurs anonymes, dont les corrections et suggestions ont grandement contribué à la clarification de mes arguments et à l'amélioration du texte.

1. Chavannes $1905: 563$.

2. De récentes exceptions doivent être signalées, notamment Altenburger 1981, Bao 2004, Chan 2008, Felt 2014 et Hüsemann 2017. Pour une liste de travaux sur le Shuijing zhu en langues chinoise et japonaise, voir Chen 2003: 11-269, et Hüsemann 2014 pour les langues occidentales.

3. Will 1992: 16; Loewe 2009: 83-84.

4. Voir Tan 1982-1988 ainsi que les volumes consacrés aux traités géographiques des Histoires dynastiques (Zhou 2006; Hu 2006).
} 
et la construction de l'espace en Chine ancienne ont connu de nombreux développements depuis les travaux pionniers de Marcel Granet ${ }^{5}$.

À partir d'une étude du Shuijing zhu, cet article présente une analyse du développement de la littérature géographique chinoise du haut Moyen Âge, élaborée à travers le thème du «décentrement» du regard géographique. Par «décentrement», il faut entendre le détachement d'une vision de l'organisation terrestre fondée sur la position centrale des symboles du pouvoir impérial. Après avoir présenté l'auteur et son œuvre, je m'attacherai à déterminer les facteurs ayant favorisé un tel décentrement dans le Shuijing zhu: facteurs religieux (essor du bouddhisme) et politique (fragmentation territoriale) d'une part, et leurs implications formelles de l'autre. Celles-ci se manifestent par le choix de commenter un texte se présentant comme un inventaire des cours d'eau du monde connu, et par la précision des descriptions hydrographiques fournies.

\section{L'auteur et son œuvre}

Le Shuijing zhu est l'œuvre de Li Daoyuan, un lettré chinois qui vécut au tournant des $\mathrm{V}^{\mathrm{e}}$ et $\mathrm{Vl}^{\mathrm{e}}$ siècles de notre ère à la cour des Wei du Nord (386534), une dynastie d'origine tabghatche. Son œuvre ébauche une synthèse inédite entre un espace aux dimensions continentales et le milieu humain qui le façonne. Les traces laissées par l'homme peuvent être matérielles (travaux, villes, monastères, stèles) ou immatérielles (histoires, récits, mythes). Euvre singulière, aussi bien technique que littéraire, le Shuijing zhu en est le témoignage tantôt direct lorsque l'auteur s'est déplacé pour observer et rendre compte (il s'est rendu des plaines mongoles jusqu'au fleuve Bleu, et du Shandong au Shaanxi), tantôt indirect lorsqu'il s'appuie sur des récits et documents tiers.

La vie de Li Daoyuan nous est essentiellement connue par deux biographies tirées du Wei shu 魏書 (Histoire des Wei) et du Bei shi 北史 (Histoire [des dynasties] du Nord), ainsi que par certaines informations qu'il distille lui-même dans le Shuijing $z h u^{6}$. La première biographie est insérée dans la catégorie des 《fonctionnaires tyranniques» ( $k u l i$ 酷吏) par Wei Shou 魏收 (506-572), tandis que la seconde, œuvre de Li Dashi 李大師 (570-628) et Li Yanshou 李延壽 (618-676), est placée à la suite de la biographie de son père, Li Fan 麗範7. Elle est sensiblement plus détaillée. Li Daoyuan naît entre 465 et 472 dans l'actuelle province du Shandong, au sein d'une famille originaire du Henan. Dès son plus jeune âge, il suit son père Li Fan, au gré des affectations de ce fonctionnaire qui fut notamment gouverneur du Qingzhou 青州 (actuel Shandong). Li Daoyuan entre ensuite dans la fonction publique et sa carrière est jalonnée d'autant de promotions que de disgrâces. Son intransigeance tout comme les vertus éducatives

5. On pourra se reporter à Granet 1934, Unno 1983, Major 1984, Cheng 1993, Chittick 1997, Lewis 2006, Puett 2009, ou encore Dorofeeva-Lichtmann 2011.

6. Par exemple, Li Daoyuan indique dans le Shuijing zhu être né à Dongqi 東齊, à côté du mont Pi 䢃, près de l'actuelle Qingzhou 青州 dans le Shandong, et non à Zhuo 溷, une localité proche de Pékin, comme l'indiquent ses biographies officielles. Voir Shuijing zhu shu, 12.1115-1116, 26.2237. 7. Voir Wei shu, 89.1925-1926; Bei shi, 27.994-996. Hüsemann (2017: 27-47) consacre un chapitre entier aux éléments biographiques de Li Daoyuan. 
de son action sont mises en valeur dans ses deux biographies. Il est assassiné en 527 par des sbires à la solde du transfuge Xiao Baoyin 蕭寶寅 $(† 530)^{8}$.

Le Shuijing zhu se présente comme le commentaire d'un bref texte anonyme, le Shuijing 水經, dont la paternité a longtemps été attribuée à Sang Qin 桑欽, un lettré des Han orientaux. Le traité bibliographique du Sui shu 隋書 (Histoire des Sui) nous apprend que «les Itinéraires des cours d'eau en trois parties ont été commentés par Guo Pu» (水經三卷郭璞注), et c'est vraisemblablement à partir de cette version que Li Daoyuan aurait rédigé ses propres notes, entre 515 et $523^{10}$.

Le texte du Shuijing zhu est divisé en quarante juan 卷 (chapitres). Dix-neuf d'entre eux sont consacrés au domaine du fleuve Jaune et à la Chine du Nord, douze juan aux bassins de la Han et de la Huai, et neuf aux régions méridionales, du fleuve Bleu jusqu'au Grand Sud, qui comprend la rivière des Perles. Ce découpage résulte d'une réorganisation du texte suite à la perte de cinq juan ${ }^{11}$.

Si les descriptions laissent la part belle à ses propres relevés pour les régions septentrionales, Li Daoyuan fait un usage particulièrement abondant de matériaux littéraires - il mentionne environ quatre cents textes, sans compter de très nombreuses stèles ${ }^{12}-$, a fortiori lorsqu'il décrit les régions méridionales où il n'est pas allé. C'est d'ailleurs ce qu'il note dans sa préface, justifiant le recours à de nombreuses sources afin de pallier le déficit d'informations dans les anciennes descriptions géographiques ${ }^{13}$.

Dans les siècles qui suivent sa composition, l'ouvrage connaît une fortune éditoriale et interprétative. Entre les Song et les Ming, la seule étude identifiée est celle de Cai Gui 蔡珪 (†1174), auteur d'un Buzheng Shuijing 補正水經 (Corrections apportées aux Itinéraires des cours d'eau) ${ }^{14}$. Le Shuijing zhu est ensuite l'objet sous les Ming, puis plus particulièrement sous les Qing, d'intenses travaux philologiques visant à séparer les «Itinéraires» de leurs

8. Prince impérial des Qi du Sud, Xiao Baoyin trouve refuge chez les Wei du Nord lorsque les Liang prennent le pouvoir en 502 et font assassiner l'ensemble de sa famille. Il bénéficie dans un premier temps de l'appui de la cour des Wei et guerroie contre les Liang, mais fait ensuite défection et se proclame empereur des Qi en 528, à Chang’an. Il est rapidement chassé, et meurt, poussé au suicide en 530. Voir Nan Qi shu, 50.864-865; Nan shi, 44.1121-1122.

9. Xin Tang shu, 58.1504; Song shi, 204.5152; Bielenstein 1993; Hüsemann 2017: 49-55.

10. Sui shu, 33.982. Guo Pu y glose vingt-six cours d'eau, du fleuve Liao 遼 au nord à la rivière des Perles au sud, et concentre davantage son commentaire sur leurs cours que sur les montagnes qui les bordent (Zhou 1990: 84).

11. Compilé durant la première moitié du XI ${ }^{\mathrm{e}}$ siècle principalement par Wang Yaochen 王堯臣 (1003-1058), le Chongwen zongmu 崇文總目 (Catalogue de la bibliothèque [de l'Institut] de la vénération de la culture), indique déjà que le Shuijing zhu a perdu cinq juan. Toutefois, les réimpressions d'exégètes Song ont permis un réarrangement des chapitres qui aboutit à un nombre de quarante juan, dans la plus ancienne édition «complète» aujourd'hui conservée, celle du Yongle dadian 永樂大典 (Grand compendium de [1'ère] Yongle), compilée au début du XV siècle. Voir Qinding Siku quanshu zongmu (zhengliben), p. 945 et Hüsemann 2017: 71-79.

12. Quatre cent trente-sept textes selon Hou Renzhi (Hou 1963: 97). Yan Gengwang mentionne quatre cent trente-six ouvrages cités (Yan 1985: 146), auxquels il faudrait ajouter de nombreux autres ouvrages non nommés. Hüsemann (2017) en dénombre trois cent quatre-vingt-quatorze. Sur les stèles, voir Shi 1987, qui en dénombre deux cent soixante-dix-huit.

13. Shuijing zhu shu, «préface», 1-2.

14. Zheng 1981: 72; Hüsemann 2017: 75 . 
«Annotations», ainsi que d'études relevant de l'exégèse épistémologique, littéraire et bien évidemment géographique. Zhu Mowei 朱謨㙔 (†1624) aurait été le premier à identifier les parties de manuscrits transmis qui composent l'ouvrage ${ }^{15}$. Son Shuijing zhu jian 水經注箋 (Notes aux Annotations des Itinéraires des cours d'eau), a permis de corriger un grand nombre d'erreurs factuelles et d'interpolations introduites par les aléas de la transmission textuelle. Il sert de base au travail des lettrés Qing, qui fondent les études «liologiques» (lixue 麗學, d'après le patronyme de Li Daoyuan), à la faveur d'un intérêt combiné pour les études géographiques et la critique textuelle. Au XVII ${ }^{\mathrm{e}}$ siècle, des savants comme le géographe Gu Zuyu 顧祖禹 (1631-1692) se penchent sur le Shuijing zhu en s'appuyant sur les progrès des connaissances géographiques. Au XVIII ${ }^{\mathrm{e}}$ siècle, les plus éminents lettrés s'y étant intéressés sont Quan Zuwang 全祖望 (17051755), Zhao Yiqing 趙一清 (1711-1764) et Dai Zhen 戴震 $(1724-1777)^{16}$.

\section{Un contexte politique et religieux propice au décentrement du regard géographique}

À la fin de l'Antiquité, le décentrement du regard géographique est en partie lié à l'ancrage du bouddhisme en Chine. Un tel déplacement est particulièrement manifeste dans le Shuijing zhu, un ouvrage composé alors que l'espace politique chinois est bipartite. Durant le Iv ${ }^{\mathrm{e}}$ siècle, le Nord de la Chine est ravagé par des conflits entre populations non chinoises qui affectent paysans comme citadins. Ils provoquent un déplacement massif de la population vers le Sud ainsi que le transfert graduel puis définitif de la cour et des élites dans une nouvelle capitale, Jiankang 建康 (actuelle Nankin), qui devient le centre politique d'un territoire s'étendant officiellement du bassin du fleuve Bleu au Vietnam actuel. Au nord, ces luttes aboutissent à la prise du pouvoir par un peuple à l'origine nomade: les Tabghatchs ou Tuoba 拓跋. Leur dynastie des Wei du Nord, officiellement fondée en 386, marque le retour à un certain calme. Le contrôle des Tabghatchs, qui se sinisent alors, s'étend sur presque toute la société chinoise ${ }^{17}$. Au Nord comme au Sud, différents régimes se succèdent jusqu'à ce qu'un puissant général des Zhou du Nord, Yang Jian 楊堅 (541-604), fonde la dynastie des Sui (581-618). Huit ans plus tard, il « unifie» l'ensemble du territoire chinois en éliminant au Sud la dynastie des Chen. Durant presque trois siècles, alors que la volonté d'unification avait été constante au Nord comme au Sud, sa concrétisation politique était demeurée impossible.

Toute cette période est marquée par l'essor du bouddhisme en Chine. À partir $\mathrm{du}_{\mathrm{III}}^{\mathrm{e}}$ siècle, la présence de cette religion se matérialise par les premières ordinations de moines chinois ${ }^{18}$ et, sous les Wei du Nord, le boud-

\footnotetext{
15. Yan 1985: 145.

16. Hu Shih 胡適 (1891-1962) a d'ailleurs tenté de comprendre comment trois grands commentateurs ayant travaillé indépendamment ont pu arriver peu ou prou à des conclusions similaires. Voir Qing shi gao, 146.4297-4298; Hu 1944 : 970-982; Hüsemann 2017 : 80-94.

17. Wright 1979: 50-51.

18. Zürcher 1990b: 182.
} 
dhisme devient religion d'État, car il procure aux souverains tabghatchs d'origine non chinoise « un fondement spirituel et une légitimité politique hors des valeurs chinoises traditionnelles ${ }^{19} \gg$. Au Sud, il est d'abord l'apanage des grandes familles, avant de connaître son apogée politique et culturel sous le règne de l'empereur $\mathrm{Wu}$ 武 des Liang (r. 502-549).

La sinisation du bouddhisme intervient durant cette longue période, comme l'attestent l'iconographie et les nombreuses traductions de soutras depuis le sanscrit. Porté par des traductions qui permettent transposition et appropriation des textes issus du bouddhisme indien, ce processus est essentiel dans la vie religieuse et intellectuelle. Il doit être interprété comme un facteur majeur des changements de perspectives dans le monde du savoir, du fait de la création d'écoles proprement chinoises d'enseignement doctrinal, et de l'instauration de cultes et de pèlerinages à l'intérieur même du territoire chinois, en particulier sur des montagnes que les moines bouddhistes s'approprient en éliminant d'anciens cultes locaux ${ }^{20}$.

Même si on a pu parler de «conquête» de la Chine par le bouddhisme, il est manifeste que les bouddhistes chinois durent lutter longtemps pour imposer leur doctrine, leurs opposants voyant en effet dans la tradition savante chinoise un horizon indépassable ${ }^{21}$. Il leur a donc fallu faire preuve d'habileté, en acceptant par exemple cette prééminence de la pensée chinoise, et proposer la fiction d'une sinisation du bouddhisme antérieure à son arrivée effective dans le monde chinois. Dans le domaine de la littérature géographique, la conséquence fut de remettre en question non pas la dualité entre centre et périphérie, mais bien leurs référents respectifs. À côté de traductions d'écrits du bouddhisme indien, certains textes bouddhiques chinois, volontairement polémiques, introduisirent un décentrement géographique inédit pour l'époque. On le trouve notamment dans le Lihuo lun 理惑論 (Doutes levés) de Mouzi 牟子 (né en 170), le Luoyang qielan ji 洛陽伽藍記 (Mémoire sur les monastères bouddhiques de Luoyang) de Yang Xuanzhi 楊街之 ( $f l . \mathrm{vI}^{\mathrm{e}}$ siècle), ou dans les écrits des moines voyageurs, comme le Foguo ji 佛國記 (Mémoire sur les pays bouddhiques) de Faxian 法顯 (337422) ou ceux de Xuanzang 玄焋 (602-664). Dans ces textes, la Chine n'est plus le centre du monde. Ils suggèrent un basculement, un renversement, en faisant de la Chine tantôt une périphérie de l'Inde, placée dès lors au centre du tableau, tantôt un centre équivalent mais parallèle au monde indien ${ }^{22}$.

En somme, après avoir pensé la construction d'un nouveau monde au cours des Royaumes combattants, après avoir unifié ce monde pendant les premières dynasties, comment pouvait-on le réorganiser pour le relever de ses ruines? Une partie de la réponse reposait sur le détour «indien», qui obligeait à décentrer le regard religieux, politique, puis géographique. Tout d'abord, la vision qu'un lettré chinois pouvait avoir de l'espace politique et

19. Cheng 1997: 365 .

20. Robson 2009: 1353, 1367; Lagerwey 2009: 401-402; Hureau 2009: 431.

21. Zürcher 1959: 264-269.

22. Sur la force du prosélytisme bouddhique durant le haut Moyen Âge, voir Zürcher 1990a. 
social avait évolué du fait de la partition entre le Nord et le Sud. L'univers religieux ainsi que les référents intellectuels n'étaient plus les mêmes, en raison de l'essor du bouddhisme notamment. Ensuite, dans une période de troubles et de division spatiale, la volonté d'établir une continuité avec un passé classique et idéalisé se conjuguait avec la nécessité de rendre compatibles la tradition scripturaire chinoise et le bouddhisme. Enfin, cette tradition savante ne dépendait plus obligatoirement de textes officiels ou de commandes impériales: un ouvrage pouvait résulter d'une initiative individuelle ${ }^{23}$.

Comment ces auteurs ont-ils procédé afin de renverser la position centrale de la Chine? Ils ont ôté toute connotation spatiale de centralité aux substantifs désignant la Chine, en supprimant le caractère zhong 中 «centre»; ils leur ont adjoint des attributs évoquant son caractère oriental en ajoutant des qualificatifs propres à centraliser les dénominations de l'Inde. On réduisait la Chine au nom de la dynastie en place, ou on lui attribuait même des noms d'origine indienne: dans le Luoyang qielan ji, Yang Xuanzhi l'appelle Dongtu 東土, les «Terres orientales ${ }^{24} »$. Pour Mouzi, la Chine ne peut plus être le centre du monde, car: "Si [le Bouddha] est né en Inde, c'est que là est le centre du monde et de la terre et le siège de l'équilibre et de l'harmonie» (所以生天䇥者 · 天地之中處其中和也); de ce point de vue, «il n'est pas certain que la terre de Han soit le centre du ciel» (漢地未必為天中也) $)^{25}$. Écrit par un laïc versé dans l'étude des textes confucéens, le Lihuo lun de Mouzi est d'un point de vue rhétorique une œuvre incontestablement chinoise ${ }^{26}$. Son auteur fonde son argumentation non pas sur une enquête ni sur une révélation à base de «faits » hypothétiques, mais sur une interprétation des Classiques. Quant à Faxian, sujet de l'empire chinois se présentant comme originaire des «marches» (biandi 邊地) ${ }^{27}$, les raisons qui lui font désigner l'Inde comme «royaume du Milieu » relèvent de la tempérance sociale, des saisons, des climats et de la nature, de son autonomie économique et agraire: «Le peuple y est riche et heureux; il n'y a ni recensement, ni règles administratives, seuls ceux qui cultivent les terres royales payent le profit de la terre. S'ils désirent s'en aller, ils s'en vont; s'ils désirent rester, ils restent» (人民殷樂, 無戶籍官法, 唯耕王地者乃輸地利, 欲去便去, 欲住便住); de même, «dans le royaume du Milieu, le froid et la chaleur s'équilibrent, les arbres atteignent parfois plusieurs milliers d'années et même jusqu'à dix mille ans» (中國寒暑均調, 樹木或數千歲, 乃至萬歲 $)^{28}$.

23. L'apport du bouddhisme ne se sent pas autant dans les textes officiels que dans les ouvrages personnels. Même s'il s'impose jusque dans les plus hauts rouages de l'État, un auteur mandaté ne peut s'en faire l'apologue dans les sources officielles, à l'inverse des œuvres individuelles.

24. Luoyang qielan ji jiaoshi, 1.1; Lourme 2014: 2.

25. Cité dans le Hongming ji 弘明集 (Recueil pour diffuser et éclairer [la Voie bouddhique]) de Sengyou 僧祐 (445-518): T. 2102, vol. 52, p. 1c25-26, 3c22. Cf. aussi Pelliot 1918-1919: 291, 304, et L'Haridon 2017: 10, 32.

26. Martin 2009: 899; L'Haridon 2013: 81.

27. Tian $2014: 47$.

28. T. 2085, vol. 51, p. 863a28-29; Drège 2013: 23, 56. 


\section{Depuis les flancs des Kunlun jusqu'aux rives de l'Anavatapta}

Rédigé plus tardivement, le Shuijing zhu exprime aussi ces changements. Dès le premier juan, Li Daoyuan reprend le commentaire de Faxian, qui avait attribué à la partie centrale de la plaine indo-gangétique, le Madhyadeśa, le nom de zhongguo 中國, une traduction littérale du sanscrit ${ }^{29}$. En voici la version donnée par le Shuijing zhu:

À l'ouest du fleuve [Jaune] se trouvent les royaumes de l'Inde, au sud desquels se situent les royaumes du centre. Le peuple y est riche. Dans ces royaumes du centre, l'habillement et la nourriture sont les mêmes que ceux de [nos] royaumes du centre, c'est la raison pour laquelle ils sont ainsi nommés.

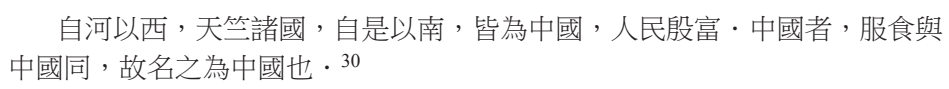

自河以西, 天竺諸國, 自是以南, 皆為中國, 人民殷富 - 中國者, 服食與 中國同，故名之為中國也. 30

Ailleurs, Faxian nomme la Chine la «Terre des [gens de] Han» (Han $t u$ 漢土) ou «des [gens de] Qin» (Qin tu 秦土) ${ }^{31}$. La traduction de zhongguo appelle deux explications. Premièrement, le fait qu'un auteur chinois l'emploie à l'endroit d'un autre monde démontre qu'il n'était pas un terme si répandu ni fréquemment utilisé pour désigner la Chine durant la période de division ${ }^{32}$. Ensuite, la possibilité existe pour un auteur chinois comme Faxian - dont la relation de voyage indique assez que sa foi bouddhique était assumée, voire revendiquée - de penser un autre centre au monde que celui de l'écoumène chinois.

Le passage précédent apparait dans le premier chapitre du Shuijing zhu qui porte sur le fleuve Jaune, et plus particulièrement sur les monts Kunlun 崦崙. Ce site, constitué de fleuves et de monts, est considéré comme l'origine à la fois spatiale et culturelle de la culture chinoise: il est le lieu de retrait de Fuxi 伏羲 et de Nüwa 女媧, deux des Trois augustes de la haute Antiquité. En traitant de l'origine des fleuves et du premier d'entre eux, le fleuve Jaune, Li Daoyuan s'interroge sur les origines de la civilisation chinoise à travers les Kunlun, considérés depuis les Han comme le centre légendaire du monde chinois (le centre politique étant matérialisé par la capitale impériale). La possibilité d'un autre centre culturel, hors de la sphère d'influence chinoise, est affirmée et justifiée par la reconnaissance de l'Inde comme espace réunissant des conditions analogues. En Inde du Nord, les données naturelles comme les facteurs culturels sont propices et ressemblent à ceux qui ont permis le développement de la civilisation chinoise. De plus, le «Kunlun indien », le lac Anavatapta, offre également en tant qu'élément lacustre une origine hydrologique qui complète avantageusement l'ensemble ${ }^{33}$ :

29. Mori 1970: 101-125; Mather 1992: 2; Wilkinson 2012: 192; Drège 2013: XIX, 2.

30. Shuijing zhu shu, 1.20; Petech 1950: 20.

31. T. 2085, vol. 51, p. 864c01, 865c25.

32. À l'époque préimpériale, zhongguo ne désigne pas une «Chine» qui n'existe pas en tant que telle, mais bien les États ou royaumes de la Plaine centrale, par opposition aux régions et aux peuples extérieurs.

33. Felt 2014: 398. 
Désormais, nous savons que les monts Kunlun sont le Tertre-sanschaleur. Comment alors affirmer qu'il puisse encore être un État barbare extérieur? J'ai examiné les propos des bouddhistes, aucun ne constitue une preuve convaincante [de cette extériorité].

而今以後, 乃知崑崙山為無熱丘, 何云乃胡國外乎? 余考釋氏之言, 未 為佳證. 34

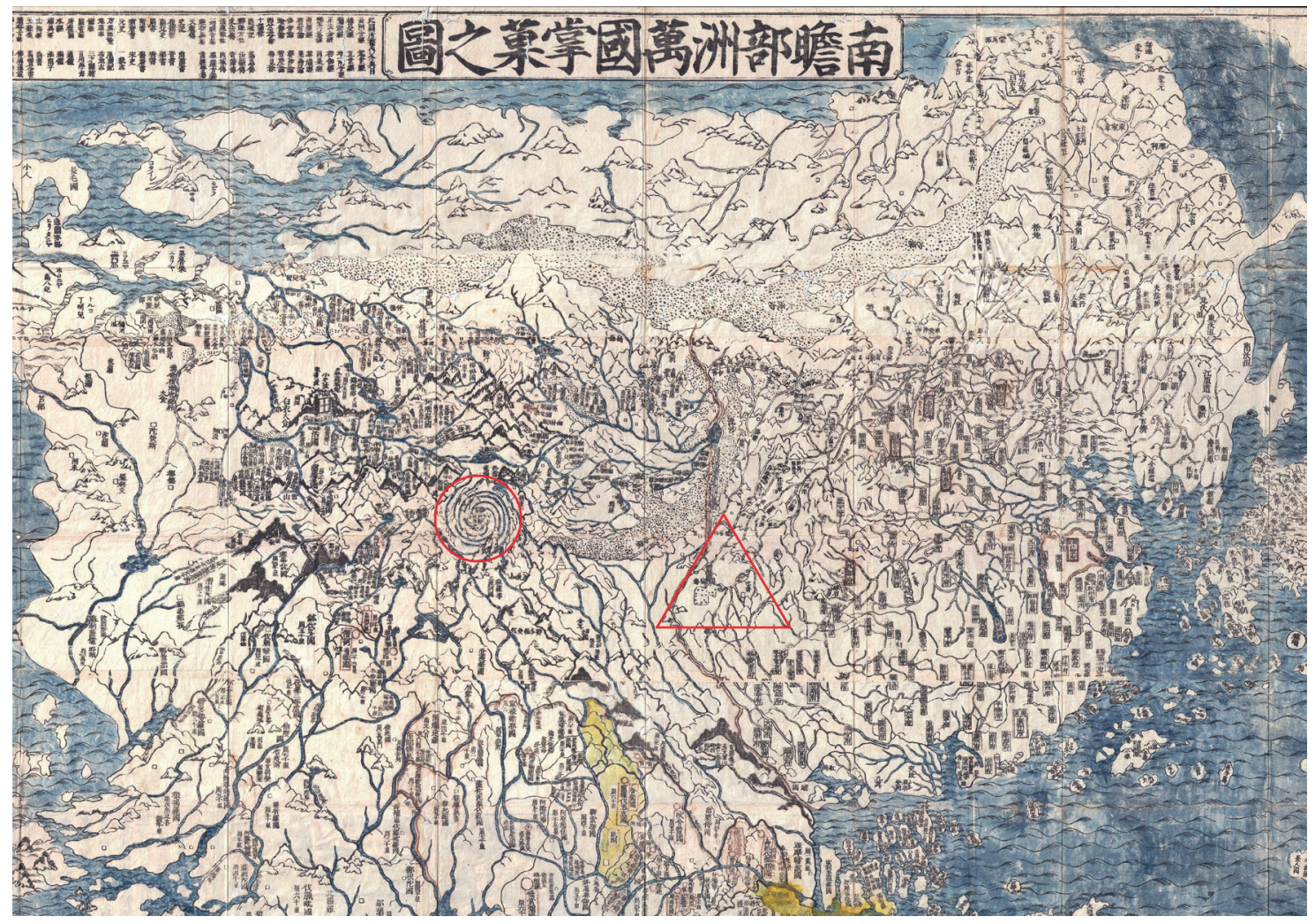

Fig. 1 - Reproduction (détail) de la Nansenbushū bankoku shōka no zu 南瞻部洲萬國掌菒之圖 (Carte de la myriade des pays du Jambūdvipa tenant en main comme un fruit), imprimée pour la première fois en 1710 et œuvre du moine Rōkashi 浪華子 (1654-1738). Devenue par la suite un modèle pour les cartes cosmographiques bouddhiques, elle présente en outre les premières influences de la cartographie européenne. Source iconographique: wikimedia commons. Voir également Unno 1994 : 428-429; je remercie Masato Hasegawa pour son aide dans l'interprétation du titre. Représenté par un tourbillon (cercle rouge), l'Anavatapta apparaît en son centre, tandis que les Kunlun (triangle rouge), dont l'emplacement exact fut longtemps un problème, soit qu'ils fussent confondus avec la source du fleuve Jaune ou parfois à partir des Song carrément omis des représentations cartographiques, sont ici figurés (bien que non nommés) autour du lac Xingxiu 星宿 (mansion-de-l'astre). Sur les déplacements cartographiques des Kunlun et leurs disparitions temporaires, voir Dorofeeva-Lichtmann 2012: 17-25.

34. Shuijing zhu shu, 1.58-59. 
Dans sa description de l'Inde, Li Daoyuan fait principalement appel aux sources bouddhiques exogènes et prête peu attention aux sources chinoises ${ }^{35}$. Il utilise donc les sources bouddhiques pour décrire les pays bouddhiques. D'une part, cela répond à une volonté revendiquée d'exploiter les sources locales les plus pertinentes pour son propos, comme la lecture d'autres chapitres le prouve. D'autre part, cela confirme l'enracinement des idées bouddhiques dans l'univers culturel chinois du haut Moyen Âge, tant ce décentrement de la Chine se traduit chez Li Daoyuan par un traitement homogène des régions de l'écoumène.

Émerge ainsi la possibilité d'un espace politique et culturel multipolaire qu'il convient de traiter dans son ensemble, en accordant autant sinon plus de place aux régions périphériques qu'au centre. Cette évolution se reflète dans le Shuijing zhu, en premier lieu car il est l'un des seuls ouvrages clairement géographiques à avoir été transmis dans sa quasi totalité pour la période. D'autres auteurs ont sûrement procédé de la même manière, si bien qu'on ne peut conclure à l'influence particulière ou au caractère novateur de l'œuvre de Li Daoyuan. En second lieu, l'importance prescriptive d'un texte comme le Shuijing zhu est évidente et se matérialise clairement dans les écrits géographiques des Tang et des Song. Ce parti pris se retrouve notamment dans la façon dont sont agencés les traités géographiques, comme celui du Sui $s h u$, ou dans une monographie impériale comme le Taiping huanyu ji 太平 寰宇記 (Géographie universelle de l'ère Taiping).

En somme, un tel «décentrement» du regard géographique est le corollaire des profondes mutations politiques et du déplacement du centre de gravité du monde chinois vers le Sud. Il trouve un écho inédit dans un ouvrage comme le Shuijing zhu qui est irrigué par l'apport des conceptions bouddhiques indiennes: ces dernières, en introduisant la possibilité d'un empire du Milieu qui ne soit plus le centre du monde, modifient la manière dont l'espace y est représenté. Les auteurs chinois abandonnent dès lors l'idée d'un centre unique entouré de périphéries: la Chine n'est plus le centre du monde, mais l'un des centres du monde.

\section{Un décentrement permis par l'architecture du texte}

À partir du VII ${ }^{\mathrm{e}}$ siècle, le Shuijing zhu est classé en tant qu'ouvrage géographique dans les histoires dynastiques ${ }^{36}$. Premier auteur à avoir proposé une synthèse historique de la géographie chinoise au $\mathrm{Xx}^{\mathrm{e}}$ siècle, Wang Yong 王庸 (1900-1956) considère l'œuvre de Li Daoyuan comme la plus importante somme d'informations géographiques avant les Sui et il la rattache à la

\footnotetext{
35. Petech 1950: 3 .

36. Cf. Sui shu, 33.984, Jiu Tang shu, 46.2014, Xin Tang shu, 58.1504, Song shi, 204.5152. Après le Song shi 宋史 (Histoire des Song), les deux seules histoires contenant un catalogue bibliographique sont le Ming shi 明史 (Histoire des Ming), qui ne recense que les ouvrages composés sous la dynastie régnante, et le Qing shi gao 清史稿 (Ébauche de l'histoire des Qing), qui comprend dans la catégorie «histoire» une sous-catégorie géographique arrangée thématiquement (Ming shi, 97.2405-2419, Qing shi gao, 146.4287-4288).
} 
sous-catégorie géographique des «Notes avec cartes sur les voies fluviales» (shuidao tuji 水道圖記) ${ }^{37}$. À l'aune des classements bibliographiques impériaux et au regard des fonctions attribuées à la géographie chinoise par la recherche contemporaine ${ }^{38}$, le Shuijing zhu relève bien de la catégorie traditionnelle «géographie» (dili 地理). Dans les classements disciplinaires du savoir en Chine, la géographie est en effet considérée comme une sous-catégorie de l' "Histoire» (shi 史), elle-même l'une des quatre branches du savoir, avec les «Classiques» (jing 經), les «Maîtres à penser» ( $z i$ 子) et les «CEuvres littéraires» ( $j i$ 集).

Le Shuijing zhu ne découpe pas l'espace géographique en fonction des frontières de l'Empire mais en prenant les cours d'eau pour unité de base. À première vue, sa structure semble en effet régie par les divisions géologiques de l'espace: les cours d'eau structurent l'ouvrage, tandis que les montagnes bornent les rivières entre elles ${ }^{39}$. Symboliquement, le caractère pervasif de l'eau permet à Li Daoyuan de couvrir l'espace du monde entier, et justifie qu'il se penche aussi bien sur les régions et localités que les cours d'eau traversent, que sur l'Empire dans son ensemble. Cet abandon du cadre de la géographie institutionnelle et administrative est conforté par une géographie davantage soumise aux critères propres à la topographie physique. Dans la préface que Li Daoyuan consacre à l'eau, élément cosmologique primordial, celle-ci est associée au Nord, à l'hiver et à la mort, mais encore à ce qui est humide, mouillé, froid, et descendant; lorsqu'elle provient du Ciel, elle recouvre, pénètre et féconde l'ensemble des éléments terrestres, et ses vertus propitiatoires favorisent l'agriculture ${ }^{40}$.

Le premier niveau de découpage spatial dans le Shuijing zhu, en fonction des fleuves, est une trame à première vue imposée par le précédent que constitue le Shuijing. Or, si le texte des «Itinéraires», le Shuijing, est organisé autour de la description des cours d'eau, depuis leur source jusqu'à leur embouchure, ses repères principaux étaient les unités administratives (les commanderies et les districts dont dépendait la description) $)^{41}$. À l'inverse, Li Daoyuan se défait dans son commentaire du poids politique des frontières et du cadre des provinces antiques: la transition entre la description d'un cours d'eau et d'un autre s'articule autour d'une description d'un lieu-dit,

37. Wang 1938: 151-152.

38. Aspect pratique du guide et de l'itinéraire, versant pseudo-scientifique et charge religieuse (Soymié 1956: 1-2), ou fonctions politique, religieuse et économique (Mathieu 1982: 130, 136, 140).

39. Selon Mark Edward Lewis (2006: 245-305), dans un texte du $\mathrm{I}^{\text {er }}$ siècle av. J.-C. comme le Shanhai jing, le «Shanjing» 山經 («Itinéraires des monts») servirait à structurer la relation verticale entre le Ciel et les hommes; en présentant un modèle horizontal rappelant une grille, le «Haijing» 海經 («Itinéraires des mers ») opposerait quant à lui le centre à la périphérie, la civilisation à la barbarie. Véhiculé par la cosmologie des Cinq agents, dont le but est d'imposer un principe régulateur d'ordonnancement du monde, le lien entre ces deux modèles serait alors matérialisé, après le domptage primordial des inondations dû à Yu le Grand, par le cours d'eau, objet mouvant et source de régulation, qui relie monts et mers. 40. Shuijing zhu shu, «préface», 1; Kalinowski 1991: 57-74. Les inondations d'un côté, qui sont un dérèglement de l'écoulement des eaux, et l'eau comme agent purificateur des mauvaises actions de l'autre, prouvent que l'élément aquatique est aussi un symbole constituant de l'harmonie sociale, et sa représentation géographique est une métaphore (et parfois une critique) du pouvoir impérial (Schaberg 1999: 185-186). Plus tard, durant le premier siècle des Ming, considéré comme un âge d'or, «l'harmonie sociale et l'ordre hydraulique vont de pair» (Will 1980: 271).

41. Hüsemann 2017: 55-57. 
fondée principalement sur des référents hydrographiques et orographiques. Ainsi de la source de la rivière Xiang 湘 (province actuelle du Guangxi), décrite en rapport avec la montagne dont elle provient. Il écrit:

La rivière Xiang sourd depuis les monts Yanghai, qui sont les monts Yangshuo. Ying Shao ( $f$. II ${ }^{\mathrm{e}}$ siècle) a écrit [dans le Compendium des us et coutumes] que «la Xiang sourd des monts Lingling», sans doute l'autre nom des [monts Yangshuo].

陽海山, 即陽朔山也・應劭曰：湘出零陵山, 蓋山之殊名也 $\cdot 42$

À l'autre extrémité de la rivière Xiang, l'embouchure est déterminée par une confluence hydrographique et les nombreux bras morts qui en résultent:

Le lieu où les trois cours d'eau se rejoignent est aussi appelé bouche Sanjiang (des-trois-fleuves). Les montagnes alentour s'ouvrent sur une passe appelée Shelie (chasse), et face au nord il y a la bouche Yang. L'une et l'autre forment le confluent de la Xiang. Leurs eaux sont d'un vert singulier et elles se jettent au nord-est dans le grand Fleuve. Les eaux de l'une sont pures tandis que celles de l'autre sont troublées, et leur confluent est appelé Jianghui (union-des-fleuves).

\section{三水所會, 亦或謂之三江口矣 ·夾山列關, 謂之射獵, 又北對養口, 咸湘 浦也・水色青異, 東北人于大江, 有清濁之別, 謂之江會也 43}

Comme on le voit, point d'indication administrative ou historique. Dans la plupart des cas, le binôme fleuve/montagne constitue le double référent spatial privilégié par l'auteur, avant qu'il ne se réfère aux marqueurs administratifs. L'approche de Li Daoyuan est une manière d'occulter les évolutions du régime impérial et de ses frontières, subordonnées avant tout aux limites physiques. Si le cadre imposé par l'auteur est celui des formes naturelles du paysage, le mode opératoire qu'il choisit est celui de l'itinéraire. Contrairement aux traités géographiques qui retracent chronologiquement l'évolution d'un lieu depuis sa fondation jusqu'à la dynastie concernée, le Shuijing zhu suit, en tant qu'itinéraire, le cours d'un fleuve. Déroulant le fil de sa progression spatiale, l'auteur s'arrête à chaque lieu important, procède à des sauts dans le temps, au mépris apparent d'une suite logique et chronologique des événements, car c'est le cours d'eau qui préside à la hiérarchie des informations qu'il fournit.

Contrairement au Shuijing zhu, les traités géographiques rédigés à la même époque présentent des bornes historiques qui renvoient à la construction politique de l'espace administratif. Sous les Qi du Sud (479-502), Shen Yue 沈約 (441-513) souhaite remettre l'espace en ordre en réorganisant, dans le Song shu 宋書 (Histoire des [Liu] Song), les données relatives à l'administration des provinces, glanées dans des sources plus anciennes comme le Han shu 漢書 (Histoire des Han) ou directement auprès des inspecteurs et des gouverneurs provinciaux ${ }^{44}$. Au siècle suivant, Xiao Zixian 蕭子顯

42. Shuijing zhu shu, 38.3120. Les caractères et les traductions en gras correspondent au texte du Shuijing.

43. Shuijing zhu shu, 38.3164-3165.

44. Song shu, 35.1027-1028. 
(489-537) propose dans le Nan Qi shu 南齊書 (Histoire des Qi du Sud) une évolution diachronique des circonscriptions administratives provinciales et de leurs hommes forts ${ }^{45}$. Nulle part les linéaments naturels ne sont mis en avant, à l'exception des références d'usage au «Yu gong» 禹貢 (《Tribut de $\mathrm{Yu}$ »), un chapitre traitant de l'ordonnancement du monde dans le Shangshu 尚書 (Classique des documents).

Les bornes administratives sont évidemment présentes dans le Shuijing zhu. Toutefois, elles le sont en tant que repères chronologiques permettant de situer le cours d'eau dans le temps, à travers les dates d'institution (des commanderies ou des royaumes), et en tant que marqueurs spatiaux, par leur localisation. Le modèle est alors le suivant: «tel fleuve sourd/coule/traverse à la frontière de tel district» (X水出/流/過X縣界). L'innovation apportée par un tel découpage a ainsi des répercussions à la fois sur l'économie générale de l'ouvrage et conséquemment sur la vision de l'auteur, comme on l'a vu à travers la préface du Shuijing zhu, qui place le texte sous le signe de l'eau et non de l'empire.

À l'inverse, les autres préfaces des textes géographiques composées durant le haut Moyen Âge insistent principalement sur le récit de la construction d'un espace politique. C'est par exemple le cas du Kuodi zhi 括地志 (Traité de toutes les régions), dont la préface se contente de résumer les modifications administratives (changements dynastiques et nominations de fonctionnaires) survenues depuis Cao Cao 曹操 (155-220):

Suite à la pacification du Wu par le Jin au cours de l'ère Taikang, le monde fut unifié. En pacifiant le Wu, [le Jin] obtint les quatre provinces de Jiao, Guang, Jing et Yang.

晉太康平吳之後, 天下一統・平吳得四州, 交廣荊揚也. 46

Décrivant une période plus ancienne, la préface du Yuanhe junxian tuzhi 元和郡縣圖志 (Traité illustré des commanderies et districts de l'ère Yuanhe) marque une rupture claire entre les souverains de l'Antiquité, dont l'objectif était la mise en place d'une civilisation, et les premiers empires des Qin et des Han, pour lesquels la domination impériale passait par l'instauration d'un système administratif de contrôle à l'échelle régionale :

Lorsque le [Premier] empereur de Qin mit les six royaumes sous sa coupe, les marquisats furent abolis et les préfectures instaurées. [L'empereur] Wu des Han écrasa les Cent Man. Profitant alors de moyens importants, il opta pour l'emploi de la force militaire [au détriment de la négociation]. Bien qu'il surpassât les Shang et les Zhou dans le nombre des districts et commanderies, son domaine d'influence et sa capacité à soumettre [les peuples] à sa majesté ne dépassait pas celles des Trois dynasties [des Xia, des Shang et des Zhou].

及秦皇並六國, 則罷侯而置守・漢武討百蠻, 則窮兵而黷武・雖裂為郡縣 者遠過於殷、周, 而教令之所行, 威懷之所服, 亦不越於三代. 47

45. Nan Qi shu, 14-15.

46. Kuodi zhi jijiao, 2.

47. Yuanhe junxian tuzhi, 1 . 


\section{Implications du décentrement dans l'approche documentaire : sources locales et témoignage personnel}

Formellement, le Shuijing zhu est-il un itinéraire topographique, un commentaire encyclopédique, un répertoire de mirabilia ${ }^{48}$ ? La taille de l'ouvrage, le nombre de ses références et la manière dont sont convoquées ces sources posent la question de sa nature, sinon encyclopédique, du moins cumulative ${ }^{49}$. Le texte de Li Daoyuan est bien un commentaire, un ensemble d'annotations (le $z h u$ 注 du titre) et non un ouvrage organisé dès l'origine par l'auteur. Il part d'une trame, d'un modèle (le Shuijing), qu'il organise selon sa logique propre. S'il est bien une œuvre personnelle et non un ouvrage géographique commandé par le pouvoir impérial, où l'auteur puise-t-il le contenu de descriptions hydrographiques souvent très détaillées? Dans des rapports de fonctionnaires locaux, dans les traités géographiques, par le bouche à oreille, suite à ses propres enquêtes? Tout cela à la fois. Si Li Daoyuan n'a pas vraiment pu franchir le fleuve Bleu pour dépeindre les sites et les fleuves méridionaux ${ }^{50}$, c'est en raison de ses affectations successives, elles-mêmes conséquences indirectes de la partition de l'Empire. Lorsque les connaissances empiriques lui font défaut, ou lorsqu'il complète des sources déjà utilisées, il convoque des mythes et des légendes, des témoignages variés et certains aspects du folklore populaire pour offrir un portrait aussi vivant que possible d'un endroit qu'il connaît $\mathrm{peu}^{51}$. Ainsi de la rencontre entre l'empereur Wen 文 (r. 424-453) des Liu Song (420-479) et un dragon, qui témoigne de sa vivacité de ton pour recréer un épisode passé:

Dans la deuxième année de l'ère Jingping des Song (424), on accueillit l'empereur Wen à Jiangling. C'est [à la bouche Jiabu 駕部 (ministère des armées)] qu'une partie de l'escorte impériale stationna. Voilà pourquoi ce lieu fut nommé ainsi. L'escorte principale de l'empereur Wen partit de Jiangling, et lorsqu'elle arriva à cet endroit, un dragon noir bondissant apparut et souleva l'embarcation dans laquelle se trouvait l'empereur. Sa suite blêmit de peur. Alors l'empereur s'adressa à son Grand annaliste Wang Tanshou en ces termes: «Si c'est ainsi que Yu des Xia a reçu son mandat du Ciel, de quelle vertu puis-je donc me prévaloir?»C'est là l'origine de l'appellation de la «Grotte du dragon», Longxue.

\footnotetext{
宋景平二年, 迎文帝于江陵, 法駕頓此, 因以為名・文帝車駕發江陵, 至 此黑龍躍出, 負帝所乘舟, 左右失色・上謂長史王曼首日乃夏禹所以受天命 矣, 我何德以堪之·故有龍穴之名焉. 52
}

48. Dans une importante étude consacrée au Linyi 林邑 (territoire actuel du Vietnam), Rolf Stein estime que Li Daoyuan était animé d'un vif intérêt pour le miraculeux, le religieux, le folklorique, et le sociologique. Des faits qu'il serait possible de qualifier d'historiques, mais que Stein juge en réalité «héroïques»: espaces sacrés, produits rares et extraordinaires, coutumes étranges, apparitions, légendes, en un mot, des mirabilia. Cf. Stein 1947: 2-3.

49. Dans la Chine classique, l'encyclopédisme se caractérise par son aspect cumulatif et par l'absence de distinction entre réel et imaginaire dans les informations fournies (Drège 2008: 335).

50. Ni d'ailleurs les plus septentrionaux (Chen 1987: 1).

51. Yan 1985: 146.

52. Shuijing zhu shu, 35.2888-2889. 
L'événement est marquant, car il concerne l'accession à la dignité impériale d'un futur empereur, résidant dans sa jeunesse dans la province de Jing 荆 53 . Li Daoyuan masque son ignorance du lieu en empruntant des détails à des sources écrites - ici le Song shu, une histoire dynastique - et orales $^{54}$. Le poids du récit, légendaire et prophétique, de l'apparition d'un dragon, signe de l'alternance entre désordre et ordre, est un symbole connu de l'avènement de tout nouvel empereur. Couplés à une autre référence à Yu le Grand ${ }^{55}$, ces éléments confèrent une autorité certaine à son discours. Si une reconstitution de l'histoire d'un personnage célèbre ou d'un mouvement est possible, elle reste toutefois subordonnée à l'espace et se réalise en fonction d'un lieu donné traversé par un cours d'eau: dans l'anecdote le personnage lui-même n'est pas évoqué autrement qu'à travers le rapport qu'il entretient avec le lieu.

Plus que de l'historiographie impériale officielle, le Shuijing zhu s'inspire davantage des ouvrages d'histoire locale et régionale, qui résultent souvent d'initiatives privées. Il n'est pas soumis aux coupes et ne se couvre pas davantage du vernis officiel des textes qui furent plus tard produits sous les Tang par les Bureaux de l'histoire car, sous les Wei du Nord et de l'Est, ces offices n'existaient pas. Même si les textes les plus cités sont les histoires, comme le Han shu (trois cent quarante-quatre mentions), ou les Classiques, comme le Chunqiu 春秋 (deux cent quarante-et-une références), les citations les plus significatives proviennent des écrits d'origine locale; ceux-ci constituent la matière principale des anecdotes comme des indications spatiales fournies par l'auteur ${ }^{56}$. L'emploi de ces écrits par Li Daoyuan découle de sa volonté de traiter uniformément de l'écoumène dans son ensemble.

Qui prétend à une œuvre personnelle doit se nommer, et s'adresser à son lectorat. L'auteur des Mémoires historiques (Shiji 史記), Sima Qian 司馬遷 (145-86 av. J.-C.), parsème son texte d'allusions à ses enquêtes et emploie souvent le style direct ${ }^{57}$. Pour valoriser son enquête, l'historien met son nom en gage d'authenticité - ce n'est pas l'œuvre d'un faussaire -, et de recherche de véracité. Les propos qui sont les siens sont crédibles puisqu'il les revendique en tant qu'auteur, et cette responsabilité assumée nourrit la crédibilité des faits exposés tout au long de l'ouvrage. Le témoignage personnel de Li Daoyuan combine ou juxtapose deux actions : d'une part, un jugement de l'auteur confrontant les sources et impliquant la réutilisation de travaux précédents et, d'autre part, une enquête de terrain annoncée par

53. Le règne long et prospère de l'empereur Wen débuta en 424 après l'assassinat de ses deux frères. Il était gouverneur de Jing lorsqu'il obtint la charge impériale (ainsi qu'au moment de cet épisode). 54. Une variante présentant un dragon jaune se trouve dans la biographie de Wang Tanshou 王 曇首 (394-430), membre du clan des Wang de Langye et conseiller important de l'empereur Wen (Song shu, 63.1678-1680). Il existe une autre version de l'épisode du dragon noir lié à la prise de pouvoir de l'empereur Wen dans le Nan shi, 2.37-38.

55. Shuijing zhu shu, 35.2877 .

56. Hüsemann 2017: 151; Lycas 2018: 47.

57. C'est d'ailleurs particulièrement manifeste dans la postface qu'il consacre au traité portant sur le «Fleuve et ses canaux» («Hequ shu» 河渠書), et dans laquelle il n'hésite pas à mettre en scène son enquête (cf. Shiji, 29.1415; Nylan 1998: 207). 
l'emploi de «moi», 《je» $(y u \text { 余, 餘 })^{58}$. Ses déplacements dans l'espace relèvent d'une forme d' "observation", chère à Marc Bloch, mais qui ne saurait être réduite au seul enregistrement des faits ${ }^{59}$.

À part Sima Qian et après lui, aucun historien - cela est différent pour les poètes - n'éprouve le besoin de quitter les murs du Bureau de l'histoire pour mener une enquête: on se nourrit de rapports de fonctionnaires et de sources plus anciennes. Historien papivore dans l'essentiel des parties qu'il consacre aux confins du monde chinois, Li Daoyuan s'appuie sur les nombreux ouvrages locaux qu'il cite nommément la plupart du temps. Ses interventions au discours direct agissent comme autant d'interpolations qui balisent le déroulement de son propos, grâce à des marqueurs spatiaux et temporels. L'observation peut se transformer alors en une «vision d'ensemble». Une telle approche, développée au sujet du «voyageur casanier ${ }^{60} »$, peut servir à analyser les raisons pour lesquelles Li Daoyuan ne s'est pas rendu dans certains lieux. En effet, ce n'est pas parce qu'il ne fait pas à chaque chapitre œuvre de découverte que son récit n'en est pas moins informé. Sans se présenter comme un explorateur, il apporte à notre connaissance des lieux éloignés du pouvoir par son utilisation raisonnée et sélective de la documentation locale dont il dispose et qui lui permet de nourrir sa vision d'ensemble.

\section{Précision hydrographique et observations littéraires}

«Dans les écrits, il convient de s'assurer que les données géographiques sont justes 》 (文章地理必須㖤當) ${ }^{61}$. Comme l'indique cette citation du Yan shi jiaxun 顏氏家訓 (Instructions familiales du clan Yan) de Yan Zhitui 顏之 推 (531-591), quelle que soit l'approche critique envisagée, la recherche de l'exactitude dans les données topographiques est souvent prônée en Chine traditionnelle. Prolongements des deux tendances de la tradition de l'école de géographie historique chinoise, la critique textuelle (kaozheng 考證) de la fin de l'Empire et la critique scientifique des spécialistes actuels de la géographie historique chinoise ont eu et ont tendance à se focaliser sur les inexactitudes topographiques des premiers textes géographiques, au premier rang desquels se situe le Shuijing zhu. Avec des moyens différents mais des objectifs analogues, les exégètes ont souvent voulu déterminer le degré d'exactitude des données présentées par l'auteur, qui peut régulièrement être «pris en défaut».

Les «erreurs factuelles» concernant les cours d'eau décrits dans le Shuijing zhu ont pour la première fois été relevées par Huang Zongxi 黃宗羲 (1610-1695) dans son Jin Shuijing 今水經 ([Annotations] actuelles des Itinéraires des cours d'eau). Leur portée est cependant à relativiser, tant $\mathrm{Li}$

\footnotetext{
58. Mori 1970: 206-207; Yan 1985: 146.

59. Ricœur $1955: 25$.

60. Pierre Bayard propose une synthèse utile de la question (Bayard 2012: 74).

61. Yan shi jiaxun jijie, 4.271; Wilkinson 2012: 243.
} 
Daoyuan admet volontiers son ignorance à de nombreuses reprises. En ce qui concerne les régions des marches méridionales, l'insuffisance potentielle de détails peut s'expliquer en premier lieu par un simple manque de données, car ces régions étaient peu ou mal contrôlées. Paradoxalement, ce défaut d'informations nous renseigne en miroir sur le terrain de l'enquête et les conceptions géographiques en vigueur durant le haut Moyen Âge.

D'un point de vue strictement stylistique, Li Daoyuan utilise à la fois la prose libre et la prose parallèle (pianwen 駢文). Cependant, un bref survol de son œuvre révèle une prédilection pour cette dernière dès lors que les descriptions de paysages deviennent littéraires. De manière générale, l'auteur a tendance à utiliser des mots simples, précis et directs pour décrire monts, fleuves, faune et flore, mais use de formes plus littéraires au moment d'élaborer anecdotes et descriptions de paysages ${ }^{62}$. Comme il existe de la poésie de paysages (de «montagnes et d'eaux», shanshui 山水), la prose de Li Daoyuan serait, selon plusieurs spécialistes de son œuvre, une élégante «prose de montagnes et d'eaux» (shanshui sanwen 山水散文) ${ }^{63}$. Les aspects littéraires de la narration de Li Daoyuan correspondent à ceux du découpage habituel de la poésie de paysage, qui voit le qing 情 (la «sensibilité») succéder au jing 景 (le «paysage»). L'auteur y convoque les champs lexicaux du végétal, du spatial, et du monumental ${ }^{64}$. Un tel mélange, entre précision technique et qualités littéraires, assigne une place particulière au Shuijing zhu au sein de la production géographique, dans la mesure où l'auteur ne recherche pas toujours explicitement l'exactitude topographique dans les représentations de l'espace.

Le mouvement et la progression sont exprimés par des verbes de franchissement tels que jing 逕 et guo 過, et des adverbes comme you 又, indiquant la reprise du mouvement narratif, et donc celle d'un nouveau déplacement dans l'espace. L'ensemble du texte est marqué spatialement par des indications reprenant les points cardinaux ou la dichotomie entre extérieur (wai 外) et intérieur (nei 內), bas (xia 下) et haut (shang 上), ainsi que des verbes relatifs à l'établissement ( $z h i$ 置) de circonscriptions ou l'érection ( $l i$ 立) de monuments. Dans l'ensemble, l'emploi des termes servant à décrire le régime des rivières dénote une maîtrise du vocabulaire hydrographique: à partir du moment où le fleuve Bleu s'engage dans les plaines alluviales bordant son cours moyen, entre les deux lacs ( $h u$ 湖 ou hai 海), les anses, canaux ou bras morts ( $p u$ 浦), coudes ( $z h a$ 溠), bouches ( $k o u \square)$, récifs ( $j i$ 磯) et autres digues (bei 陂) se multiplient.

Une autre méthode relativement fructueuse pour démontrer la technicité du texte consiste à relever les hapax que l'on y trouve. Certes, d'autres textes contemporains, aujourd'hui perdus, contenaient probablement des termes d'emploi similaire, et les auteurs des dictionnaires modernes n'ont probablement pas tout lu. Cependant, ces rédacteurs tirent souvent, dans les définitions des termes qu'ils proposent, un sens à partir d'un exemple

62. Tan 1982: 5 .

63. Bao 2004: 171; Chan 2008: 145-154.

64. Voir par exemple Shuijing zhu shu, 38.3123-3125, 3132, 3139. 
originel ou unique lorsque la signification d'un terme n'apparaît pas ailleurs. Ce phénomène n'est pas propre au Shuijing zhu, mais une étude lexicale des termes relevant de la description physique et littéraire de l'espace démontre une récurrence dans leur emploi par Li Daoyuan. C'est un signe de la richesse de son texte. En outre, leur utilisation est tout autant liée à la recherche d'un vocabulaire stylisé qu'à l'emploi quasi-systématique de la prose parallèle par l'auteur lorsqu'il se livre à des descriptions littéraires. Pour la seule rivière Xiang 湘 traversant la province moderne du Hunan, les résultats sont les suivants (ceux obtenus à partir d'autres cours d'eau offrent des échantillons tout aussi significatifs):

\begin{tabular}{|l|l|l|}
\hline \multicolumn{3}{|c|}{ Tableau des hapax relatifs à la description de la rivière Xiang 湘 (juan 38) } \\
\hline Hapax & Signification & Typologie/Champ lexical \\
\hline changuo 鷹郭 & Faubourgs d'une ville & Description urbaine \\
\hline chizhu 簏竹 & Espèce de bambou & Flore \\
\hline daiyue 帶約 & Confluence de deux rivières & Hydrographie \\
\hline fulian 幅練 & Étoffe de soie blanche (métaphore des nuages) & Description de paysage \\
\hline gangling 岡嶺 & Cime d'une montagne & Orographie \\
\hline guangyuan 廣圓 & Superficie d'un lac & Hydrographie \\
\hline Mao 毦 & Hydronyme & Hydrographie \\
\hline songjie 竦傑 & $\begin{array}{l}\text { Élevé et majestueux (à propos d'une mon- } \\
\text { tagne) }\end{array}$ & $\begin{array}{l}\text { Orographie/Description } \\
\text { de paysage }\end{array}$ \\
\hline tongxing 銅腥 & $\begin{array}{l}\text { Odeur de cuivre nauséabonde (se dégageant } \\
\text { de la rivière) }\end{array}$ & Hydrographie/Description \\
\hline xiao 奫 & Eaux claires et limpides (de rivière) & Hydrographie/Description \\
\hline xiemu 寫目 & Laisser courir le regard au gré de ses pensées & Perception de l'espace \\
\hline xuanquan 旋泉 & Source tourbillonnante & Hydrographie \\
\hline zhence 枕側 & Proximité (espace entre montagne et rivière) & Hydrographie/Orographie \\
\hline zhijin 枝津 & Défluent (bras sortant d'une rivière) & Hydrographie \\
\hline
\end{tabular}

Les champs lexicaux de la description physique et de l'expression sensorielle se distinguent clairement. D'un côté, on remarque un vocabulaire technique se rapportant aux caractéristiques physiques, orographiques et fluviales; de l'autre, on relève le champ lexical de la représentation des paysages et des sensations éprouvées par l'auteur à leur vue ou perçues à leur évocation (à travers d'autres œuvres).

Comme le tableau des hapax le montre, Li Daoyuan a réellement le souci de la correction lexicale, de l'importance du mot juste. Sans que des langues ou dialectes locaux soient explicitement mentionnés, il fait grand cas de l'étymologie populaire et rend hommage à de nombreux informateurs. Parallèlement, il n'estime pas indispensable d'avoir vu pour rédiger: s'appuyant sur l'avis de tiers, il peut arriver à Li Daoyuan de ressentir des émotions propres, qu'il parviendra à extérioriser et à exprimer de lui-même ${ }^{65}$.

65. Tan 1982:3. 
La «vision d'ensemble» mentionnée dans la partie précédente à propos de la sélection textuelle ressort aussi au niveau du ressenti littéraire. Ce n'est pas parce que la géographie décrite n'est pas tangible pour l'auteur qu'elle transmet moins efficacement ses intentions.

\section{Conclusion}

Afin d'aborder la problématique de cet article, il était nécessaire de présenter d'abord l'auteur et le contexte socio-historique de son temps. En raison de la partition de l'espace politique et des bouleversements religieux qui le caractérisent, le haut Moyen Âge offrait à un auteur chinois comme Li Daoyuan des paradigmes nouveaux pour penser les configurations du monde qui était le sien. On a ensuite montré comme le décentrement du regard géographique de l'auteur était fonction de l'essor du bouddhisme et du positionnement de l'Inde en tant qu'autre centre du monde. Pour ce faire, Li Daoyuan s'est inspiré des sources bouddhiques rédigées en chinois et il a situé les origines des mondes chinois (les monts Kunlun) et indien (le lac Anavatapta) sur un pied d'égalité. La seconde partie de l'article a détaillé les dispositifs formels lui ayant permis de représenter l'espace: découpage du texte par fleuve et non par unité administrative, utilisation novatrice de la documentation locale, importance de l'enquête, et précision des descriptions techniques.

De la centaine de cours d'eau cités dans le Shuijing aux mille deux cents et quelques du Shuijing zhu, le texte de Li Daoyuan couvre l'étendue du monde connu de son temps. Il cite près de quatre cents sources et trois cents stèles. Le caractère inédit de cette division naturelle fait cohabiter l'universel et le régional. Chaque description de l'environnement physique et culturel d'une rivière peut être analysée indépendamment des autres, comme autant de monographies locales: les visées de l'ouvrage sont à l'échelle du monde, tandis que les descriptions restent locales.

Travail de géographie historique, ouvrage géographique dont l'objet est l'histoire, compendium de la mémoire culturelle de l'Empire, le Shuijing $z h u$ est une synthèse médiévale du savoir géographique et de ses branches historique, administrative et culturelle, abordée par le biais d'une étude fluviale. En se démarquant des traités techniques en rapport avec l'espace et consacrés à l'astronomie, à la géographie administrative, aux fleuves et canaux, il constitue, en plusieurs aspects, un tournant épistémologique dans l'histoire de la géographie: contrairement aux traités qui délaissent le paysage et ses caractéristiques, il s'attache aux traces matérielles du passé chinois et ouvre ainsi une voie inédite dans la représentation de l'espace en Chine. S'il s'inscrit dans une tradition historiographique déjà établie, il accompagne également le développement des écrits de type régional qui préfigurent les monographies locales, et qui émergent avec la fragmentation du pouvoir qui suit la chute des Han et renforce le poids des régionalismes. 


\section{Bibliographie}

\section{Sources primaires}

Bei shi 北史 [Histoire (des dynasties) du Nord], Li Yanshou 李延壽. Pékin, Zhonghua shuju, 1997.

Jiu Tang shu 舊唐書 [Ancienne histoire des Tang], LiU Xu 劉昫. Pékin, Zhonghua shuju, 1997.

Kuodi zhi jijiao 括地志輯校 [Traité de toutes les régions. Édition critique], LI Tai 李泰 (éd. He Cijun 賀次君). Pékin, Zhonghua shuju, 1980 (2010).

Luoyang qielan ji jiaoshi 洛陽伽藍記校釋 [Édition critique du Mémoire sur les monastères bouddhiques de Luoyang], ZHou Zumo 周祖謨 (éd.). Pékin, Zhonghua shuju, 1963 (1987).

Ming shi 明史 [Histoire des Ming], Zhang Tingyu 張廷玉 (dir.). Pékin, Zhonghua shuju, 1997.

Nan Qi shu 南齊書 [Histoire des Qi du Sud], XIAo Zixian 蕭子顯. Pékin, Zhonghua shuju, 1972.

Nan shi 南史 [Histoire (des dynasties) du Sud], Li Yanshou 李延壽. Pékin, Zhonghua shuju, 1997.

Qinding Siku quanshu zongmu (zhengliben) 欽定四庫全書總目 (整理本) [Édition palatiale du Catalogue général de la Bibliothèque des quatre trésors établie sur commande (version arrangée)], JI Yun 紀昀, LU Guangming 盧光明. Pékin, Zhonghua shuju, 1997.

Qing shi gao 清史稿 [Ébauche de l'Histoire des Qing], ZHAo Erxun 趙爾巽 (dir.). Pékin, Zhonghua shuju, 1997.

Shiji 史記 [Mémoires historiques], Sima Qian 司馬遷. Pékin, Zhonghua shuju, 1959 (1963).

Shuijing zhu shu 水經注疏 [Annotations aux Itinéraires des cours d'eau. Édition annotée], Li Daoyuan 麗道元 (éd. YANG Shoujing 楊守敬 \& XIONg Huizhen 熊會貞). Nankin, Jiangsu guji chubanshe, 1999.

Song shi 宋史 [Histoire des Song], Tuotuo 脫脫 (dir.). Pékin, Zhonghua shuju, 1997.

Song shu 宋書 [Histoire des (Liu) Song], SHEN Yue 沈約. Pékin, Zhonghua shuju, 1974.

Sui shu 隋書 [Histoire des Sui], Wei Zheng 魏徵. Pékin, Zhonghua shuju, 1973 (1987).

Taishō shinshū Daizōkyō 大正新修大藏經 [Canon bouddhique de l'ère Taishō], TAKAKUSU Junjirō 高楠順次郎 \& WATANABE Kaikyoku 渡邊 海旭 (éd.). Tokyo, Taishō issaikyō kankōkai, 1924-1935.

Wei shu 魏書 [Histoire des Wei], WeI Shou 魏收. Pékin, Zhonghua shuju, 1974 (1984).

Xin Tang shu 新唐書 [Nouvelle histoire des Tang], OuYANG Xiu 歐陽修. Pékin, Zhonghua shuju, 1997.

Yan shi jiaxun jijie 顏氏家訓集解 [Interprétations rassemblées des Instructions familiales de maître Yan], YAN Zhitui 顏之推 (éd. WANG Liqi 王利器). Shanghai, Shanghai guji chubanshe, 1986.

Yuanhe junxian tuzhi 元和郡縣圖志 [Traité et cartes des commanderies et districts de l'ère Yuanhe], Li Jifu 李吉甫. Pékin, Zhonghua shuju, 1983 (2008). 


\section{Sources secondaires}

Altenburger, Engelbert

1981 «Historische Geographie des Jangtse. Kommentar zum Shuijing $z h u »$, Thèse de doctorat, Tübingen, Eberhard Karls Universität.

BAO Yuanhang 鮑遠航

2004 «Shuijing zhu wenxian xue wenxue yanjiu» 水經注文獻學文學研究 [Recherches littéraires et philologiques sur les Annotations aux Itinéraires des cours d'eau], Thèse de doctorat, Pékin, université Shoudu shifan.

BAYARD, Pierre

2012 Comment parler des lieux où l'on n'a pas été?, Paris, Minuit. Bielenstein, Hans

1993 «Notes on the Shui Ching», Bulletin of the Museum of Far East Antiquities 65, p. 257-272.

Chan Ching-Yee (Chen Jingyi) 陳靜儀

2008 《Shuijing zhu zhangfa jiegou yu zhuanshu tese yanjiu» 水經注章 法結構與撰述特色研究 [Structures et caractéristiques narratives des Annotations aux Itinéraires des cours d'eau], Mémoire de Master, Taipei, université Shih Hsin.

Chavannes, Édouard

1905 «Les Pays d'occident d'après le Wei Lio», T'oung Pao 6, p. $519-571$.

CHEN Qiaoyi 陳橋驛

1987 Shuijing zhu yanjiu erji 水經注研究二集 [Recherches sur les Annotations aux Itinéraires des cours d'eau, tome 2], Taiyuan, Shanxi renmin chubanshe.

2003 Shuijing zhu yanjiu siji 水經注研究四集 [Recherches sur les Annotations aux Itinéraires des cours d'eau, tome 4], Hangzhou, Hangzhou chubanshe.

Cheng, Anne

1993 «La Notion d'espace dans la pensée traditionnelle chinoise», in Flora Blanchon (dir.), Aménager l'espace, Paris, Presses de l'université de Paris-Sorbonne, p. 33-44.

1997 Histoire de la pensée chinoise, Paris, Seuil.

Chiтtick, Andrew

1997 «Pride of Place. The Advent of Local History in Early Medieval China», Thèse de doctorat, Ann Arbor, University of Michigan.

Dorofeeva-Lichtmann, Vera

2011 «Spatial Organization of Ancient Chinese Texts (Preliminary Remarks) », in Karine ChemLA (dir.), History of Science, History of the Text, Dordrecht, Springer, p. 3-50.

2012 «A history of a spatial relationship: Kunlun Mountain and the Yellow River source from Chinese cosmography through to Western cartography», Circumscribere 11, p. 1-31. 
DrÈGE, Jean-Pierre

2008 «Histoire et civilisation de l'écrit en Chine. Livres de voyages», Annuaire de l'École pratique des hautes études (EPHE), Section des sciences historiques et philologiques 139, p. 334-335.

Drège, Jean-Pierre (trad.)

2013 FaXIan, Mémoire sur les pays bouddhiques, Paris, Les Belles Lettres.

FeLt, David Jonathan

2014 «Patterns of the Earth. Writing Geography in Early Medieval China», Thèse de doctorat, Stanford, Stanford University.

Granet, Marcel

1934 (1999) La Pensée chinoise, Paris, Albin Michel.

Hou Renzhi 侯仁之 (dir.)

1963 Zhongguo gudai dili mingzhu xuandu 中國古代地理名著選讀 [Choix de lectures d'œuvres remarquables en géographie de la Chine ancienne], Hongkong, Zhonghua shuju.

Hu Axiang 胡阿祥

2006 Song shu zhoujun zhi huishi 宋書州郡志匯釋 [Annotations au traité géographique du Livre des Song], Hefei, Anhui jiaoyu chubanshe.

Hu, Shih

1944 «A Note on Ch'uän Tsu-wang, Chao I-ch'ing and Tai Chên. A Study of Independent Convergence in Research as Illustrated in their Works on the Shui-ching chu», in Athur W. Hummel (dir.), Eminent Chinese of the Ch'ing Period (1644-1912), vol. II, Washington, Government Printing Office, p. 970-982.

Hureau, Sylvie

2009 «Production et dissémination de textes bouddhiques: traductions et apocryphes », in John LAGERWEY (dir.), Religion et société en Chine ancienne et médiévale, Paris, Cerf, p. 429-459.

HüsEmanN, Jörg Henning (Xu Yuehe 徐約和)

2014 «Bainian lai xifang sheji Shuijing zhu zhi yanjiu gaikuang xiaozhuan》百年來西方涉及《水經注》之研究概況小傳 [Survol de plus de cent ans de recherches occidentales sur le Shuijing zhu], Hanxue yanjiu tongxun 漢學研究通訊 33 (4), p. 19-29.

2017 Das Altertum vergegenwärtigen: Eine Studie zum Shuijing zhu des Li Daoyuan, Leipzig, Leipziger Universitätsverlag.

KaLINOWSKi, Marc

1991 Cosmologie et divination dans la Chine ancienne. Le Compendium des cinq agents (Wuxing dayi, $v I^{e}$ siècle), Paris, EFEO.

L'HARIDON, Béatrice

2013 «La controverse dans le Mouzi », Études chinoises 32 (1), p. 77-96.

L'HARIDON, Béatrice (trad.)

2017 Meou-Tseou, Dialogues pour dissiper la confusion, Paris, Les Belles Lettres. 
LAGERWEY, John

2009 «Religion et politique pendant la période de division», in John LAGERWEY (dir.), Religion et société en Chine ancienne et médiévale, Paris, Cerf, p. 397-428.

LEwIS, Mark Edward

2006 The Construction of Space in Early China, Albany, State University of New York Press.

Loewe, Michael

2009 «Knowledge of other Cultures in China's Early Empires», in Kurt A. RaAflaub \& Richard J. A. Talbert (dir.), Geography and Ethnography: Perceptions of the World in Pre-Modern Societies, Chichester, Wiley-Blackwell, p. 74-88.

LOURME, Jean-Marie (trad.)

2014 YANG Xuanzhi, Mémoire sur les monastères bouddhiques de Luoyang, Paris, Les Belles Lettres.

LyCAS, Alexis

2018 «Géographie religieuse des Six dynasties», Annuaire de l'École pratique des hautes études (EPHE), Section des sciences religieuses 125, p. 43-48.

MAJOR, John S.

1984 "The Five Phases, Magic Squares and Schematic Cosmography», in Henry Rosemont (dir.), Explorations in Early Chinese Cosmology, Chico, Scholar, p. 133-137.

MARTIN, François

2009 «Buddhism and Literature», in John Lagerwey \& Lü Pengzhi (dir.), Early Chinese Religion. Part Two: The Period of Division (220-589 AD), Leyde, Brill, p. 891-952.

MATHER, Richard B.

1992 «Chinese and Indian Perceptions of Each Other Between the First and Seventh Centuries », Journal of the American Oriental Society 112 (1), p. 1-8.

Mathieu, Rémi

1982 «Fonctions et moyens de la géographie dans la Chine ancienne», Asiatische Studien 36 (2), p. 125-152.

MoRI Shikazō 森鹿三

1970 Tōyoggaku kenkyū : Rekishi chirihen 東洋學研究: 歷史地理篇 [Recherches en études orientales: textes de géographie historique], Kyoto, Dōhō.

NYLAN, Michael

1998 «Sima Qian: A True Historian?», Early China 24, p. 203-246.

Pelliot, Paul

1918-1919 «'Meou-tseu ou les doutes levés', traduit et annoté», T'oung Pao 19 (5), p. 255-433.

Petech, Luciano

1950 Northern India according to the 'Shui-ching-chu', Rome, Istituto italiano per il Medio ed Estremo Oriente. 
PuetT, Michael J.

2009 «Centering the Realm: Wang Mang, the Zhouli, and Early Chinese Statecraft», in Benjamin A. Elman \& Martin Kern (dir.), Statecraft and Classical Learning: The Rituals of Zhou in East Asian History, Leyde, Brill, p. 129-154.

Riceur, Paul

1955 (1964) Histoire et vérité, Paris, Seuil.

RoBson, James

2009 «Buddhist Sacred Geography», in John Lagerwey \& LÜ Pengzhi (dir.), Early Chinese Religion. Part Two: The Period of Division (220-589 AD), Leyde, Brill, p. 1353-1398.

SchaberG, David

1999 «Travel, Geography, and the Imperial Imagination in Fifth Century Athens and Han China», Comparative Literature 51 (2), p. 152-191.

SHI Zhicun 施執存

1987 Shuijing zhu beilu 水經注碑錄 [Catalogue des stèles des Annotations aux Itinéraires des cours d'eau], Tianjin, Tianjin guji chubanshe.

Soymié, Michel

1956 «Le Lo-feou chan: étude de géographie religieuse», Bulletin de l'École française d'Extrême-Orient 48, p. 1-139.

STEIN, Rolf A.

1947 «Le Lin-yi, sa localisation, sa contribution à la formation du Champa et ses liens avec la Chine», Han-hiue, bulletin du centre d'études sinologiques de Pékin 2, p. 1-335.

TAN Qixiang 譚其驤 (dir.)

1982-1988 Zhongguo lishi ditu ji 中國歷史地圖集 [Collection des cartes historiques de Chine], Pékin, Ditu chubanshe.

TIAN, Xiaofei

2014 «Tales from Borderland: Anecdotes in Early Medieval China», in Jack W. Chen \& David SchABERg (dir.), Idle Talk. Gossip and anecdote in traditional China, Berkeley, University of California Press, p. 38-54.

UnNo, Kazutaka

1983 «The Geographical Thought of the Chinese People: with Special Reference to Ideas of Terrestrial Features », Memoirs of the Research Department of the Toyo Bunko 41, p. 83-97.

1994 «Cartography in Japan», in J. B. HARLEY \& David Woodward (dir.), The History of Cartography, vol II-2. Cartography in the Traditional East and Southeast Asian Societies, Chicago, University of Chicago Press, p. 346-477.

Wang Yong 王庸

1938 (1984) Zhongguo dilixue shi 中國地理學史 [Histoire de la géographie chinoise], Shanghai, Shanghai shudian. 
WILKINSON, Endymion

2012 Chinese History: A New Manual, Cambridge, Harvard University Asia Center.

WiLL, Pierre-Étienne

1980 «Un Cycle hydraulique en Chine: la province du Hubei du XVI ${ }^{\mathrm{e}}$ au XIX ${ }^{\mathrm{e}}$ siècle», Bulletin de l'École française d'Extrême-Orient 68, p. 261-287.

1992 «Chinese Local Gazetteers: An Historical and Practical Introduction», Notes de recherche du Centre Chine [EHESS] 3, p. 7-39.

Wright, Arthur F.

1979 «The Sui Dynasty (581-617)», in Denis Twitchett (dir.), The Cambridge History of China Volume III. Sui and T'ang China, Part I, Cambridge, Cambridge University Press, p. 48-149.

YAN Gengwang 嚴耕望

1985 «Zhonggu shidai jibu zhongyao dili shu. Di yi jiang. Shuijing $z h u \gg$ 中古時代幾部重要地理書第一講水經注 [Quelques éminents ouvrages géographiques du Moyen Âge chinois I: les Annotations aux Itinéraires des cours d'eau], Hanxue yanjiu tongxun 漢學研究通訊 4 (3), p. 145-148.

ZHENG Dekun 鄭德坤

1981 《Shuijing zhu banben kao» 水經注版本考 [Analyse critique des éditions des Annotations aux Itinéraires des cours d'eau], in Zheng Dekun 鄭德坤, Zhongguo lishi dili lunwenji 中國歷史地 理論文集 [Recueil d'essais de géographie historique chinoise], Taipei, Lianjing chuban shiye, p. 65-94.

ZHоu Zhenhe 周振鶴

1990 《Zhongguo gudai zhuanxie Shuijing de chuantong»中國古代撰 寫水經的傳統 [La tradition des écrits portant sur les Itinéraires des cours d'eau en Chine ancienne], Lishi dili 歷史地理 8, p. 83-87.

2006 Han shu dili zhi huishi 漢書地理志匯釋 [Annotations au traité géographique de l'Histoire des Han], Hefei, Anhui jiaoyu chubanshe.

ZÜRCHER, Erik

1959 (1972, 2007) The Buddhist Conquest of China, Leyde, Brill.

1990a Bouddhisme, Christianisme et société chinoise, Paris, Julliard.

1990b «Han Buddhism and the Western Region» in Wilt L. IDEMA \& Erik ZÜRCHER (dir.), Thought and Law in Qin and Han China: Studies Dedicated to Anthony Hulsewé on the Occasion of His Eightieth Birthday, Leyde, Brill, p. 158-182. 